\title{
Peces asociados con la deriva de macroalgas en Aysén, Chile
}

\author{
Fishes associated to drifting macroalgae in Aysen, Chile \\ Germán Pequeño $^{1}$, Daniela Farías ${ }^{1}$, Martín Thiel ${ }^{2}$ e Iván Hinojosa ${ }^{2}$ \\ ${ }^{1}$ Instituto de Zoología “Ernst F. Kilian”, Universidad Austral de Chile \\ Casilla 567, Valdivia, Chile \\ ${ }^{2}$ Facultad de Ciencias del Mar, Universidad Católica del Norte \\ Casilla 117, Coquimbo, Chile \\ gpequeno@uach.cl
}

\begin{abstract}
Resumen.- La obtención de restos de macroalgas flotando a la deriva en aguas frente al sur de Chile permite, por primera vez, explorar la presencia de peces nadando junto con ellas. Se registra la presencia del pez aguja Leptonotus blainvilleanus (familia Syngnathidae); la cojinova Seriolella porosa (familia Centrolophidae); el pampanito, Stromateus stellatus (familia Stromateidae) y el torito Bovichtus sp. (familia Bovichthidae); este último, un ejemplar diferente de Bovichtus chilensis, única especie de ese género conocida en América del Sur. El hallazgo de representantes de esas especies permite agregar nuevos antecedentes, potencialmente importantes, para explicar aspectos de la distribución geográfica de las mismas.
\end{abstract}

Palabras clave: Ictiogeografía, Bovichtus, Leptonotus, Seriolella, Stromateus

\section{Introducción}

Desde hace algunos años, la literatura ictiológica ha mostrado la importancia de las macroalgas flotantes, que prestan cobertura y un verdadero medio de transporte para muchas formas de peces (Fedoryako 1982 y 1989, Mitchell \& Hunter 1970). Tales algas generalmente de gran tamaño, son arrancadas del sustrato por diferentes acciones mecánicas, generalmente naturales y empiezan a derivar arrastradas por las corrientes superficiales y los vientos. Tal fenómeno permite que peces, comúnmente de hábitos litorales o vinculados vitalmente a las orillas y las profundidades someras, se cobijen bajo estas algas y así naden debajo o junto con ellas. Puede tratarse de ejemplares adultos, pero aparentemente la gran mayoría son formas juveniles y de pequeño tamaño. En esos restos de algas flotantes también se encuentran otros organismos, como por ejemplo pequeños crustáceos, moluscos y otros invertebrados, que a su vez constituyen una fuente alimentaria para los peces acompañantes. En el fondo, se encuentra allí un

\begin{abstract}
The obtention of floating macroalgae debris in waters of southern Chile, for the first time allowed to explore the presence of fishes swimming together with such kind of ensemble. The presence of the following three species was recorded: the pipefish Leptonotus blainvilleanus (Family Syngnathidae), the chicy ruff Seriolella porosa (Family Centrolophidae); the starry butterfish Stromateus stellatus (Family Stromateidae) and the thornfish Bovichtus sp. (Family Bovichtidae). The last one, a specimen different from Bovichthus chilensis, the only species of the genus known in South America. The finding of representatives of these species allowed the addition of new antecedents, potentially important to explain aspects of their geographical distribution.
\end{abstract}

Key words: Ichthyogeography, Bovichtus, Leptonotus, Seriolella, Stromateus

conjunto vivo derivando, con potencialidad de llegar exitosamente a otras costas e iniciar posibles colonizaciones, lo cual reviste importancia para los estudios de ictiogeografía, entre otros aspectos.

Las macroalgas constituyen un hábitat de gran importancia para muchas especies litorales, las que además de protegerse de depredadores, también se alimentan, como fuera observado y estudiado tempranamente en la costa de California (Feder et al. 1974, North \& Hubbs 1968). Las algas son arrancadas del fondo debido a la acción de un fuerte oleaje u otra acción mecánica, arrastrando consigo una multitud de pequeños invertebrados que las pueblan, sirviendo de alimento a los peces de su entorno.

Un proyecto llevado a cabo en la zona de Aysén (Chile), destinado al estudio de algas a la deriva ha proporcionado la oportunidad de capturar y estudiar una pequeña muestra de peces que en ellas se cobijaban. Nuestro objetivo es conocer la composición taxonómica de la misma y revisar los alcances biogeográficos de su presencia en ese ambiente flotante. 


\section{Material y métodos}

Los peces fueron capturados con redes lanzadas desde la cubierta del buque científico "VIDAL GORMAZ", en las siguientes estaciones de colecta de la segunda etapa del Crucero CIMAR 8 - Fiordos: bahía Ana Pink, No 4 (45082'60”S, 74\%89'64”W), 20 nov. 2002; canal

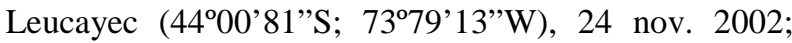

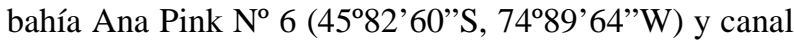

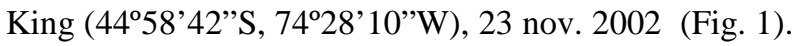
Una vez en cubierta, los ejemplares fueron fijados en formalina al $10 \%$ y así, llevados hasta el Laboratorio de Ictiología de la Universidad Austral de Chile, en
Valdivia. Allí se les trasladó a alcohol de $70^{\circ}$, para facilitar su estudio. La determinación taxonómica se hizo usando bibliografía especializada (Bravo 1997, Bravo et al. 1999, Cassia \& García de la Rosa 1994, Fritszche 1980, Haedrich 1967, Haedrich \& Horn 1972, McDowall 1980 y 1982, Pequeño \& Sáez 2004). Las mediciones morfológicas, así como las cuentas merísticas, se hicieron sobre la base de las mencionadas publicaciones. Con el fin de facilitar el trabajo de identificación taxonómica, se trabajó con el siguiente material comparativo, depositado en la colección de peces marinos de la Universidad Austral de Chile:



Figura 1

Estaciones de recolección de los peces flotando junto con Macrocystis pyrifera

Sampling stations of fishes drifting with Macrocystis pyrifera 
Leptonotus blainvilleanus (Eydoux \& Gervais 1837), IZUA-PM-1305, un ejemplar 117 mm LT, Cariquilda (Maullín, Chile), 18 agosto 1990; IZUA-PM-1307, un ejemplar, $125 \mathrm{~mm} \mathrm{LT}$, puerto Condell (cerca isla Rivero, 4538'S, 74²13'W), febrero, 1988; IZUA-PM1344, 3 ejemplares, 95 a 132 mm LT, estuario Maullín, 24 noviembre 1990.

Bovichtus chilensis (Regan, 1913), IZUA-PM-132, cuatro ejemplares 40 a $42 \mathrm{~mm}$ LE, Mehuín (costa de Valdivia), 16 enero 1968; IZUA-PM-1483, un ejemplar $47 \mathrm{~mm}$ LE, Los Liles (al sur de Corral, Valdivia), 10 febrero 1992; IZUA-PM-1732, siete ejemplares 39 a 49 mm LE, Reñaca (norte de Viña del Mar), 16 enero, 1968.

Seriolella caerulea Guichenot, 1848, IZUA-PM-1132, dos ejemplares, $76 \mathrm{~mm}$ y $117 \mathrm{~mm}$ LE, isla Robinson Crusoe (archipiélago de Juan Fernández), 28 octubre, 1984.

Seriolella porosa Guichenot, 1848, IZUA-PM-238, un ejemplar, $180 \mathrm{~mm}$ LE, Corral (Valdivia, Chile), 6 septiembre 1974.

Seriolella violacea Guichenot, 1848, IZUA-PM-11, 5 ejemplares, entre 96 y 159 mm LE, Corral-Amargos (Valdivia, Chile), 9 enero 1961.

En el texto se usan las abreviaturas siguientes: LT=longitud total; LE=longitud estándar; IZUAPM=acrónimo para la colección de peces marinos del Instituto de Zoología de la Universidad Austral de Chile (UACH).

\section{Resultados}

El análisis de los ejemplares permitió reconocer a un ejemplar del orden Syngnathiformes, familia Syngnathidae, correspondiente a la especie Leptonotus blainvilleanus (Eydoux \& Gervais, 1837) y ocho ejemplares del orden Perciformes. Entre estos últimos se contabilizaron un ejemplar de la familia Bovichtidae, Bovichtus sp.; seis ejemplares de la familia Centrolophidae, representantes de la especie Seriolella porosa Guichenot, 1848 y un ejemplar de la familia Stromateidae, de la especie Stromateus stellatus Cuvier, 1829. El total de la muestra fue de nueve ejemplares. En todos los casos, estos ejemplares acompañaban a los restos flotantes de Macrocystis pyrifera.

El ejemplar de Leptonotus blainvilleanus, de 8,8 mm LT, correspondió a un individuo aparentemente hembra, aún juvenil, pero grande dentro de ese estado. Fue capturado en la estación del canal King (Fig. 1). Su presencia en toda la cosa de Chile ha sido señalada desde antaño (Cárdenas \& Pequeño 1990, Fritzsche 1980, Mann 1954), por lo que no constituye novedad alguna en la lejana localidad de captura. Las características del individuo son coincidentes con las anotadas en la literatura.

El ejemplar de Bovichtus sp. de $42,7 \mathrm{~mm}$ LE, correspondió a un individuo juvenil pronto a alcanzar la madurez. Fue capturado en la estación de Ana Pink $\mathrm{N}^{\mathrm{o}}$ 6. Mostró claramente diferencias morfológicas externas que lo diferencian de la conocida especie Bovichtus chilensis (Regan, 1913), al ser comparados ejemplares de similar tamaño (Bravo 1997, Pequeño \& Inzunza 1987) (Fig. 2). El ejemplar presentó los siguientes índices morfométricos, en \% de la LE: longitud de la base de la aleta dorsal, 18,5; longitud de la base de la aleta anal, 37,2; diámetro del ojo, 7,7; distancia preanal, 53,9; distancia pre-dorsal, 23,9; distancia prepélvica, 20,4; longitud aletas pélvicas, 23,7; longitud aletas pectorales, 26,7; longitud maxilar + premaxilar, 7,3; altura mínima del pedúnculo caudal, 7,5; longitud de la cabeza, 24,8; distancia post-ocular, 13,1; distancia pre-ocular, 4,4 y altura máxima del cuerpo, 22,5. Primera aleta dorsal con 8 espinas, segunda dorsal con 20 radios, anal con 15 radios, pectoral 15 radios. El color del ejemplar difiere de lo conocido mediante la literatura, dado que es de color azulado parejo en el dorso, tornándose más claro hacia los costados, para terminar prácticamente blanco en el vientre. Los principales rasgos morfológicos, especialmente la longitud de la cabeza, fuertemente influida por las espinas operculares, contribuyeron a determinar que la especie a la cual pertenece el ejemplar es diferente de Bovichtus chilensis. Sin embargo, es prematuro adscribirla a especies con características muy similares, como es el caso de Bovichtus angustifrons Regan 1913, que habita aguas de Australia y Tasmania. Entre las características diferenciales que exigen su estudio más detenido, especialmente con otros ejemplares que se pudiese obtener, está el diámetro del ojo que constituye el 31,1\% de la LC, así como el último radio de la aleta anal, que no alcanza la base del primer rayo procurrente de la caudal.

Los seis ejemplares de Seriolella porosa tuvieron tamaños entre 12,6 y 30,1 mm LE, es decir, individuos juveniles chicos. El ejemplar mayor fue capturado en la estación del canal Leucayec, en tanto que los otros cinco, fueron capturados en la estación Ana Pink N ${ }^{\circ} 4$. Pese a su pequeño tamaño, sus características fueron coincidentes con las descripciones de la especie y discriminadas de otras similares (Haedrich 1967, Haedrich \& Horn 1972, McDowall 1980 y 1982, 
Stehmann \& Lenz 1973). Seriolella porosa ha sido considerada como propia de la región y su captura, desde el punto de vista ictiogeográfico, no constituye mayor novedad.

El único ejemplar de Stromateus stellatus es un juvenil de 23,8 mm LE. Es de tamaño bastante pequeño, pero sus características morfológicas, especialmente la ausencia de aleta pélvica, pero reteniendo las características del suborden Stromateoidei y de la familia Stromateidae, conducen a su reconocimiento taxonómico. S. stellatus es la única especie de la familia Stromateidae conocida en aguas chilenas (Haedrich 1967, Haedrich \& Horn 1972, Horn 1973, Pequeño 1989). Las características de estados de vida iniciales de otros peces del mismo suborden permitieron descartar la pertenencia de este ejemplar a otras especies del Pacífico oriental y Atlántico sur occidental (Ahlstrom et al. 1976, Cassia \& García de la Rosa 1994).

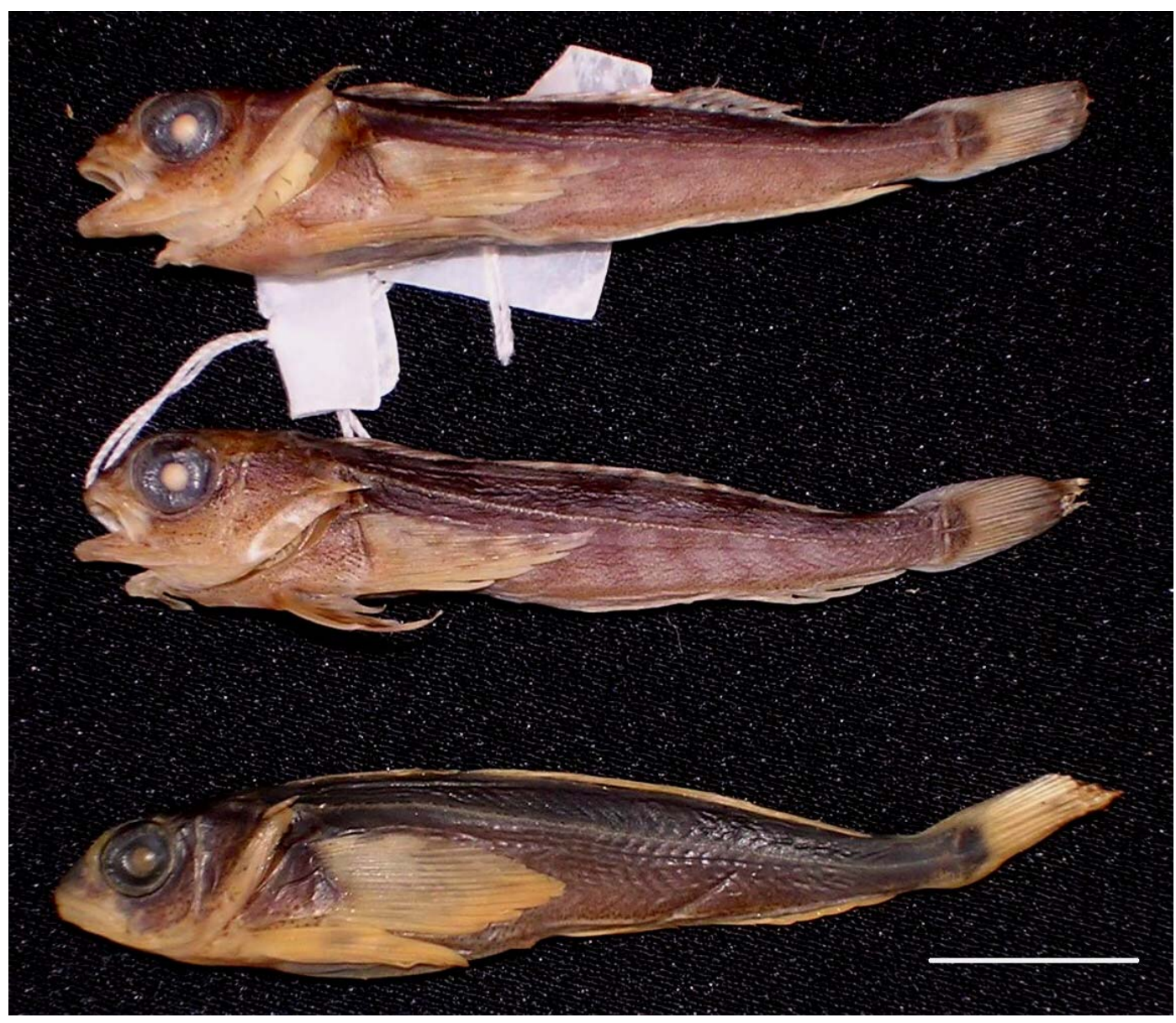

Figura 2

Visión comparativa de dos ejemplares de Bovichtus chilensis, IZUA-PM-132 (los dos de arriba) y uno de Bovichtus sp., IZUAPM-2314 (abajo). Escala: 1 cm

Comparative vision of two specimens of Bovichtus chilensis, IZUA-PM-132 (the two uppermost) and one of Bovichtus sp., IZUA-PM2314 (below). Scale: $1 \mathrm{~cm}$ 


\section{Discusión}

La separación de grandes algas desde el sustrato, como lo es Macrocystis pyrifera, suele ser un fenómeno frecuente en las costas de Chile y, es por ello que tales restos se encuentran a menudo varados en las playas. Tal fenómeno, generalmente causado por marejadas y tempestades, conduce a que esos restos de algas deriven de acuerdo con el impulso del viento o las corrientes marinas, siendo acompañadas en su recorrido por numerosos organismos que interactúan a modo de pequeñas comunidades que se autoabastecen entre sí, al amparo que les brinda la masa de algas que les cobija. También es un fenómeno conocido, que muchas especies tienen parte importante su ciclo vital en las zonas intermareal y submareal superior, justamente donde se desarrollan las algas. No es extraño entonces que, al salir éstas flotando a la deriva, muchos de aquellos peces litorales salgan de su ambiente original también con ellas e inicien un recorrido que puede ser de corto o largo alcance, contribuyendo al repoblamiento o colonización de nuevos lugares. Este fenómeno puede tener consecuencias para la distribución geográfica de muchas especies. En general, no es un fenómeno nuevo puesto que Fedoryako (1982) y Mitchell \& Hunter (1970), entre otros, ya lo han señalado para otras localidades geográficas, sin embargo, del océano Pacífico sur oriental, aparentemente no se conocen estudios de esta naturaleza (Fedoryako 1989).

La aguja de mar, Leptonotus blainvilleanus, ha sido reiteradamente encontrada entre algas de diversos tamaños en Chile, ya sea en estuarios o en costas abiertas y en canales australes (Cárdenas \& Pequeño 1990). Pertenece a una familia cuyo origen ha sido asociado principalmente con aguas de tipo templado a abiertamente tropicales (Fritzsche 1980). Sin embargo, las características biológicas de la especie permiten suponer que los individuos no pueden alejarse de su hábitat bentónico inter y submareal superior e incluso estuarial, sin el riesgo de exponer la vida. De hecho, $L$. blainvilleanus no se encuentra en el pélagos abierto. Pero, dentro de su particular ciclo de vida, que implica la incubación de los huevos por el macho, las larvas que éste libera tienen un período de vida pelágica litoral, muy cerca de la orilla. En ese período, las larvas pueden ser transportadas por corrientes que circulan muy cercanas a la orilla y eso, contribuye a explicar la relativamente amplia distribución geográfica que tiene la especie entre Tumbes (Perú) (Chirichigno \& Vélez 1998), el extremo sur de Chile y también la costa sur de Argentina (Cárdenas \& Pequeño 1990, Fritzsche 1980).
El hallazgo de un ejemplar de L. blainvilleanus entre los restos de macroalgas flotantes era de esperar, dado que se sabe que los individuos de esta especie han sido encontrados en praderas de macroalgas (Moreno \& Jara 1984), hallazgo que permite reconocer otro posible mecanismo de dispersión, para ejemplares de tallas mayores que las correspondientes a las fases larvarias o juveniles de otras especies, pero que están de acuerdo con otras cuya capacidad de natación es limitada como es el caso de esta especie.

En cuanto a Bovichtus sp., se trata de un solo individuo de 42,7 $\mathrm{mm}$ LE con una serie de características que permiten reconocerlo como miembro del género, pero no de la especie Bovichtus chilensis, hasta ahora la única conocida de América del Sur (Fig. 2). La longitud de la cabeza y el diámetro del ojo, se encuentran entre las características diagnósticas más importantes, que la aproximan a Bovichtus angustifrons Regan, 1913 (Bravo 1997, Bravo et al. 1999). Además, su coloración es bastante diferente de aquella de Bovichtus chilensis. Dicha coloración, según la literatura, parece ser distinta a la de los adultos (Bravo 1997). B. angustifrons fue descrita originalmente para Tasmania y Australia (Victoria, South Australia, New South Wales) (Bravo 1997, Hardy 1988, Regan 1913) y es considerada entre las formas más primitivas del suborden Notothenioidei (Voskoboinikova \& Bruce, 2001). Los peces del género Bovichtus son considerados como bentónicos, con escasa movilidad natatoria en aguas someras de costas del Hemisferio Sur. Recientemente, se ha considerado que $B$. chilensis tiene una distribución amplia para lo que se conoce de su género, que va desde Coquimbo, Chile $\left(30^{\circ} \mathrm{S}, 73^{\prime} \mathrm{W}\right)$ hasta Marimeli, también en Chile $\left(41^{\circ} 42^{\prime} \mathrm{S}, 72^{\circ} 27^{\prime} \mathrm{W}\right)$ y en Argentina, desde la Patagonia norte hasta Mar del Plata, incluyendo la Antártica (Bravo et al. 1999). El hallazgo de este ejemplar nominado como Bovichtus sp. sugiere mayor esfuerzo en la búsqueda de posibles ejemplares similares, para contribuir a resolver el nuevo problema que plantea su presencia.

Es necesario recordar que muchos peces del suborden Stromateoidei, entre ellos los de la familia Centrolophidae (por ej. género Schedophilus), son frecuentemente encontrados nadando bajo medusas, algo también común en otras especies del suborden Stromateoidei y que Seriolella está adscrito a la familia Centrolophidae. También en este caso, podemos suponer que esta natación bajo restos de algas a la deriva puede ser una forma de dispersión que puede haber estado operando en el hemisferio sur. De modo similar al anterior, encontramos a Stromateus stellatus, del mismo suborden, pero de la familia Stromateidae. Esta especie es común en aguas de Chile y Perú 
(Chirichigno \& Vélez 1998), principalmente en las zonas pelágica y demersal sobre la plataforma continental. Es probable que Macrocystis pyrifera constituya una alternativa de hábitat importante en su ciclo de vida, especialmente para las fases juveniles. Pero, lo más importante a lo cual apunta nuestro trabajo, es a demostrar que en esas algas a la deriva existen peces, que pueden ser trasladados $\mathrm{y}$, con ello, tener alguna influencia en la explicación de la distribución geográfica de los peces. Esta materia, en el Pacífico sur oriental, deberá ser mejor desarrollada con miras al progreso de la ictiogeografía litoral.

\section{Agradecimientos}

Se agradece a las bibliotecarias Sra. Jeanette Santana (Facultad de Ciencias del Mar, Universidad de Valparaíso) y Patricia Pacheco (Escuela de Ciencias del Mar, Pontificia Universidad Católica de Valparaíso), su colaboración en asuntos bibliográficos; don León Matamala (Instituto de Zoología, UACH), prestó valioso apoyo en el laboratorio. Estos son resultados derivados del Proyecto CIMAR 8 - Fiordos, patrocinado por el Comité Oceanográfico Nacional (CONA).

\section{Literatura citada}

Ahlstrom EH, JL Butler \& B Sumida. 1976. Pelagic stromateoid fishes (Pisces, Perciformes) of the eastern Pacific: kinds of distributions, and early life histories and observations on five of these from the northwest Atlantic. Bulletin of Marine Science 26 (3): 285-402.

Bravo R. 1997. Revisión de la familia Bovichtidae (Perciformes, Notothenioidei) y aspectos biogeográficos. Tesis Doctoral, Universitat de Barcelona, Barcelona, 388 pp.

Bravo R, D Lloris, G Pequeño \& J Rucabado. 1999. Revisión de las distintas especies del género Bovichtus (Perciformes, Bovichtidae) citadas para el cono sur americano y península Antártica. Revista de Biología Marina \& Oceanografía 34 (2): 123-137

Cárdenas T \& G Pequeño. 1990. Dimorfismo sexual, huevos y larvas de "aguja de mar" Leptonotus blainvilleanus (Eydoux y Gervais, 1837) (Osteichthyes: Syngnathidae). Biología Pesquera, Chile 19: 27-38.

Cassia MC \& SB García de la Rosa. 1994. Características diferenciales del desarrollo larval de Stromateus brasiliensis, y su distribución en el Atlántico sur occidental. Frente Marítimo, Montevideo 15: 157-162.
Chirichigno N \& J Vélez. 1998. Clave para identificar los peces marinos del Perú (Segunda edición). Instituto del Mar del Perú, Publicación Especial, 502 pp.

Feder HM, CH Turner \& C Limbaugh. 1974. Observations on fishes associated with kelp beds in southern California. Department of Fish and Game, Fish Bulletin 160: 1-144.

Fedoryako BI. 1982. Annotated list of fishes associated with drifting objects. En: PP Shirshov (ed), Unsufficiently studied fishes of the open ocean. Institute of Oceanology, pp. 110-118, Academy of Sciences of the USSR, Moscú.

Fedoryako BI. 1989. A comparative characteristic of oceanic fish assemblages associated with floating debris. Journal of Ichthyology 29 (3): 128-137.

Fritzsche RA. 1980. Revision of the eastern Pacific Syngnathidae (Pisces: Syngnathiformes), including both recent and fossil forms. Proceedings California Academy of Sciences 42 (6): 181-227.

Haedrich RL. 1967. The stromateoid fishes: systematics and a classification. Bulletin of the Museum of Comparative Zoology, Harvard 135 (2): 31-139.

Haedrich RL \& MH Horn. 1972. A key to the stromateoid fishes. Woods Hole Oceanographic Institution, Technical Reports, 1-47 (Unpublished manuscript).

Hardy G. 1988. A revisión of Bovichtus Cuvier, 1831 (Pisces: Bovichthyidae) from Australia, with description of a new deep water species from the New Zealand sub-Antarctic. Journal of Natural History 22: 1639-1655.

Horn MH. 1973. Systematic comparison of the stromateid fishes Stromateus brasiliensis (Fowler) and Stromateus stellatus (Cuvier) from coastal South America with a review of the genus. Bulletin of the British Museum of Natural History 24 (7): 319-339.

Mann G. 1954. Vida de los peces en aguas chilenas. Instituto de Investigaciones Veterinarias y Universidad de Chile, Santiago, $343 \mathrm{pp}$.

McDowall RM. 1980. Seriolella caerulea Guichenot, 1848 in New Zealand waters (Stromateoidei: Centrolophidae). Journal Royal Society of New Zealand 10 (1): 65-74.

McDowall RM. 1982. The centrolophid fishes of New Zealand (Pisces: Stromateoidei). Journal Royal Society of New Zealand 12: 103-142.

Mitchell CT \& JR Hunter. 1970. Fishes associated with drifting kelp, Macrocystis pyrifera, off the coast of southern California and northern Baja California. California Fish and Game 56 (4): 288-297. 
Moreno CA \& F Jara. 1984. Ecological studies on fish fauna associated with Macrocystis pyrifera belts in the south of Fueguian islands, Chile. Marine Ecology Progress Series 15: 99-107.

North WJ \& C Hubbs. 1968. Utilization of kelp-bed resources in southern California. Department of Fish and Game, Fish Bulletin 139: 1-264.

Pequeño G. 1989. Peces de Chile. Lista sistemática revisada y comentada. Revista de Biología Marina, Valparaíso, 24 (2): $1-132$

Pequeño G \& A Inzunza. 1987. Variabilidad intraespecífica y estatus sistemático del "torito", Bovichthys chilensis Regan, 1913 (Osteichthyes: Bovichthyidae). Boletín Sociedad Biología Concepción 58: 127-139.

Pequeño G \& S Sáez. 2004. Peces marinos comunes del litoral de Valdivia. Corporación Ambiental del Sur, Ediciones Surambiente, Santiago, 87 pp.
Regan CT. 1913. The Antarctic fishes of the Scottish National Antarctic Expedition. Transactions Royal Society of Edinburgh 49: 229-292.

Stehmann M \& M Lenz. 1973. Ergebnisse der Forschungreisen des FFS "Walther Herwig" nach Südamerika XXVI. Systematik und Verbreitung der Artengruppe - Seriolella punctata (Schneider, 1801), S. porosa Guichenot, 1848, S. bobula (Günther, 1869) sowie taxonomische Bemerkungen $\mathrm{zu}$ Hyperoglyphe Günther, 1859 und Schedophilus Cocco, 1839 (Osteichthgyes, Stromateoidei, Centrolophidae). Archiv für Fischereiwissenchaften 23 (3): 179-201.

Voskoboinikova O \& B Bruce. 2001. Development of bony skeleton during ontogeny of Bovichtus angustifrons (Bovichtyidae, Notothenioidei) with comparative notes on development of bony skeleton of congolli Pseudaphritis urvilli (Pseudaphritidae). Journal of Ichthyology 41 (1): 57-66. 